\title{
Simulation Analysis of Supply Chain System under Emergencies Based on Multi-agent Model
}

\author{
Qianying $\mathrm{Li}^{1}$, Jirong Wang ${ }^{2, *}$, Dawei Song ${ }^{3}$, Jun $\mathrm{Li}^{4}$ \\ ${ }^{1}$ Management School, Sheffield University, Sheffield, s37rw, United Kingdom \\ ${ }^{2}$ College of Mechanical and Electronic, Qingdao University, Qingdao, 266071, China \\ ${ }^{3}$ Qingdao Port International Co. LTD, Qingdao, 266011, China \\ ${ }^{4}$ College of Information Engineering, Qingdao University, Qingdao, 266071, China \\ * Corresponding author
}

\begin{abstract}
Under the framework of quantity discount contract, the coordination problems caused by emergencies in the two-echelon centralized or decentralized supply chain with a single supplier and a single retailer are discussed respectively. The dynamic models of the supply chain based on multi- agent system are established. Simulations are conducted using swarm6.0 software platform. The optimal strategies coping with the unexpected emergencies are given.
\end{abstract}

Keywords-supply chain; emergency; multi-agent; simulation; coordination strategy.

\section{INTRODUCTION}

The supply chain system often encounters a variety of unexpected emergencies. If the emergencies cannot be handled in a timely manner, huge losses will be brought to the supply chain system. Supply chain emergency coordination based on multi-agent studies the collaborations of agents, where each agent represents a node enterprise in supply chain system, so as to improve the ability to respond to emergencies and the performances of the supply chain system. Through mutual collaboration, the integrity of the supply chain is maintained and the benefits of the whole supply chain are improved. The multi-agent models of two echelon supply chain with a single supplier and a single retailer have been established by introducing multi-agent technique to supply chain management. The supply chain emergency coordination mechanisms under emergencies have been studied.

\section{II.DYNAMIC MODEL OF SUPPLY CHAIN BASED ON MULTI-AGENT}

\section{A. Problem description}

When emergencies did not occur, enterprises in supply chain make their production plans and orders according to market demands. When emergencies occur, such as earthquakes, the market scales of products will change suddenly, which will then impact the price sensitive coefficient of the products. The market demands change along with the changes of market scales and price sensitive coefficient, which would lead to the production plans of supplier and the orders of retailor inconsistent with the new actual market demands. Also, due to the changes of the external environment of supply chain caused by emergencies, such as earthquake damage to the traffic environment, intermediate links of supply chain will be influenced, which will directly lead the enterprises in supply chain to spend much more time than usual to complete the shipment or sale, and will indirectly affect the cost of the whole supply chain.

Specifically, there are two possible scenarios. The first is that the actual market demands are greater than the supplier's planned productions. In this case, the supplier needs a temporary increase in production to meet the actual market demands. Because that will be related to increase the procurement of raw materials or even to break the original production plan, some extra costs to the supply chain will be brought. The second is that the actual market demands are less than the supplier's planned productions. In this case, with a surplus of products cannot be sold on the market, the supplier will sell the excess products at a lower price in the secondary market in order to minimize the losses as much as possible, which will also bring some additional costs to the supply chain. In addition, because the profits of supply chain are affected indirectly by the changes of the external environments due to emergency, additional costs to the supply chain will also be generated.

For the influences to different parameters caused by emergencies, special notations can be used to solve the problem, when considering the profits of the supply chain, in which the sign of "+" or "-" should be paid much attention. So the two scenarios do not need to be discussed separately in establishing the model of supply chain in the above two scenarios.

Material flow, capital flow and information flow among the members, of supply chain are shown in Fig.1. 


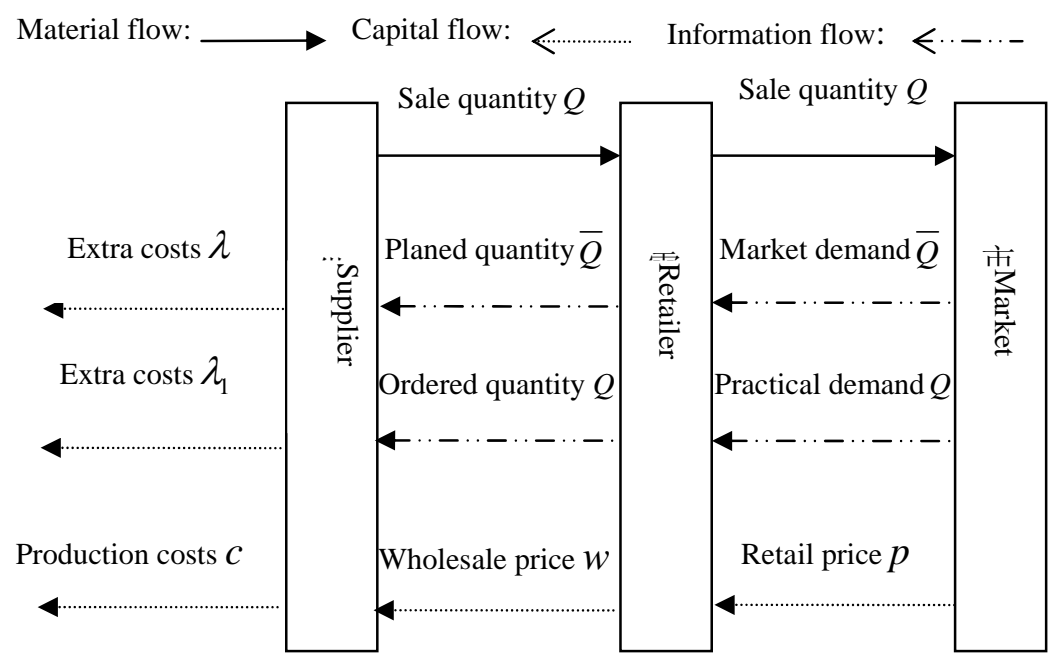

Figure 1. Flow chart of supply chain

\section{B. Multi-agent dynamic model of supply chain under emergency}

When the market scales and the price sensitivity coefficient are disturbed, the external environment of supply chain is also affected by emergency. Adopting demand driven method, the specific process of the whole supply chain can be described.

1) Agent of market demand

a) When emergencies occur, the Agent of market demand will provide new demand information $Q$. The supplier and the retailer develop their own new production plan and order quantity respectively by knowing the actual market demands.

b) For the same product, the disturbance amplitude of the new maximum market demand on the basis of the original demand is $\Delta D$, which means that the new market size becomes $D+\Delta D$.

c) The disturbance amplitude of the new price sensitivity coefficient on the basis of the original coefficient is $\Delta k$, which means that the new price sensitive coefficient becomes $k+\Delta k$.

The variables description:

$Q$ is a rational integer values between 0 and $D+\Delta D$.The relationship between $Q$ and $\bar{Q}$ is uncertain. Here only two cases are discussed without considering the equal situation.

$\Delta D$ is the disturbance amplitude of market scale, where it can be either positive or negative. Positive means the market scales caused by emergencies increase and negative indicates the market scales decrease.

$\Delta k$ is disturbance amplitude of price sensitive coefficient, where it can be either positive or negative. Positive means the price sensitive coefficient caused by emergencies increase and negative indicates the price sensitive coefficient decrease, only if $k+\Delta k>0$

2) Agent of retailer

a) The retailer's orders ordered from suppliers are determined according to the actual market demand. Because the new market demands are ${ }^{Q}$, the retailer's orders ordered from suppliers are ${ }^{Q}$ also.

b) Assume that the profit losses of the supply chain indirectly affected by external environmental changes caused by emergencies are linearly dependent on the actual market demand, and the additional unit costs are $\lambda$, the additional costs brought by are $\lambda Q$.

c) Inventory status is not considered, That is, under the new market demands, the retailer can get the ordering quantity of products from the supplier and can sale the products ordered to the market one-time, or the products ordered are all sold out on demand during the period of disturbance in the supply chain caused by emergencies, regardless of the inventory costs and consumptions.

d) The appropriate new retail price $p$ is set according to the actual market demand. The products ordered are all sold at price $p$, regardless of the consumptions.

The variables description:

$Q$ is the new order quantity of retailers, which is in line with market demand.

$\lambda$ is the additional unit costs of the supply chain which is caused by external factors as a result of emergencies. Those portions of the total additional costs are considered linearly dependent on the actual market demands $Q$.

$p$ is the new retail price which is set according to the new market demand by the retailer. And the new price can keep in a period of time until all the products sold. 


\section{3) Agent of supplier}

a) The supplier should rearrange its own production schedule based on the new market demands the retailer faced. So that, to satisfy the practical market demands, the supplier sets its new production quantities as $Q$. Then the extra costs, which are brought due to the temporary changes of the production schedule, are considered as follow:

(1) When the actual market demands $Q$ are greater than the original production plan $\bar{Q}$, and assume that the extra unit costs due to the increased additional production quantities are $\lambda_{1}$, the extra total costs arising from this situation are $\lambda_{1}(Q-\bar{Q})$.

Here mark $(x)^{+}$is introduced, and set $(x)^{+}=\max (0, x)$. Thus, the extra total costs caused by the circumstances that the actual market demands are inconsistent with the original production plans of supplier can be considered unitedly, which can be simplified to $\lambda_{1}(Q-\bar{Q})^{+}+\lambda_{2}(\bar{Q}-Q)^{+}$.

b) The unit production cost is $\mathrm{c}$.

c) The new orders of retailer can be met in a timely manner. The supply chain's cost losses resulting from external factors caused by emergencies have been discussed on the part of the Agent of retailer.

d) Surplus products can be sold at a lower price, regardless of the inventory problem.

The variables description:

$Q$ is the new production plans for suppliers. When the value of $Q$ is greater than the original production plans, the supplier will achieve the value through increasing production to meet the demands of the retailer's ordering requirements. When the value of $Q$ is smaller than the original production plans, suppliers will sell the surplus products in the secondary market to reduce costs.

$\lambda_{1}$ is the extra cost brought by the supplier's temporary increasing production, $\lambda_{1}>0$.

$\lambda_{2}$ is the extra cost brought by selling surplus products of the supplier. Because the surplus product causes losses to the supply chain, the unit cost of this part should be lower than the supplier's unit production cost, satisfy the condition $\lambda_{2}<c$.

$c$ is the unit production cost for the supplier's products, assuming that c was not impacted by emergencies.

\section{THE RESULT ANALYSIS}

The initial values of parameter of supplier agent and retailer agent are given at input interface. Assume that $\mathrm{c}=4$,

$\mathrm{a} 1=1, \mathrm{a} 2=0.5, \mathrm{D}=300, \mathrm{k}=0.5, \mathrm{a}=0.2$, the adjusted optimal production plans of supplier are shown in Fig.2 (a) and (b) when kchange is -0.1 or 0.1 , where disturbance of market scale Dchange is in [-100,100].

It can be seen through the image that the production plan is needed to adjust only when the magnitude of the disturbance reaches a certain level, otherwise, the original production plan should keep unchanged. It illustrate that there is a mutual restriction relationship between the two disturbances. An adverse impact of a disturbance can be offset or partially offset by the beneficial impact of another disturbance. And with the increase in market scale, the optimal production quantity increased. With the increase of price sensitive coefficient of the disturbance the optimal production quantity reduced.

The impacts on supply chain profit using emergency coordination strategy and maintaining the original strategy are shown in Fig.3 (a) and (b) respectively, where the market scale of Dchange is in the range $[-100,100]$, when kchange is -0.1 or 0.1 .

It can be seen through the image that the supply chain profits are much larger using emergency coordination strategy than that maintaining the original strategy.

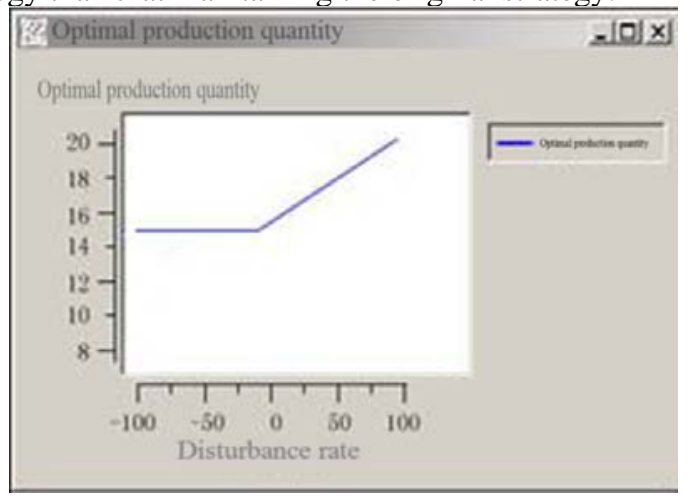

(a) kchange $=-0.1$

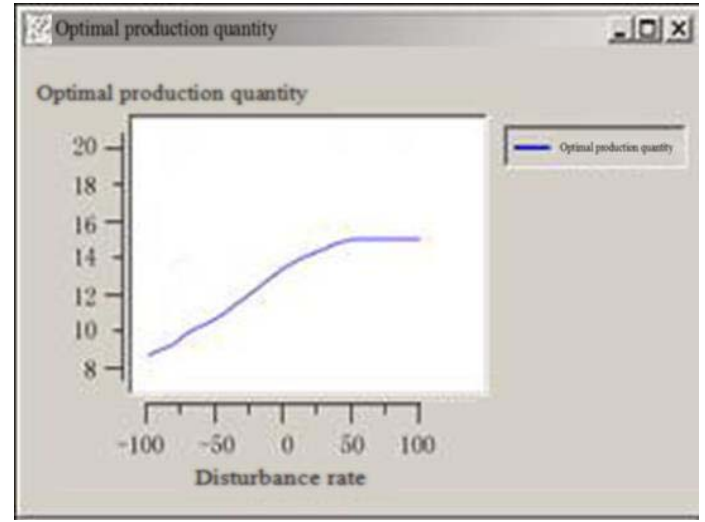

(b) kchange $=0.1$

Figure 2. The optimal production quantities of supplier after the disturbance

Compared Fig.3 with Fig.2, the change rate of supply chain profit increases with the increase of Dchange, when the optimal order quantity unchanged or increased, while decreases with the increase of kchange. When the optimal order quantity decreased, it is just the opposite. 


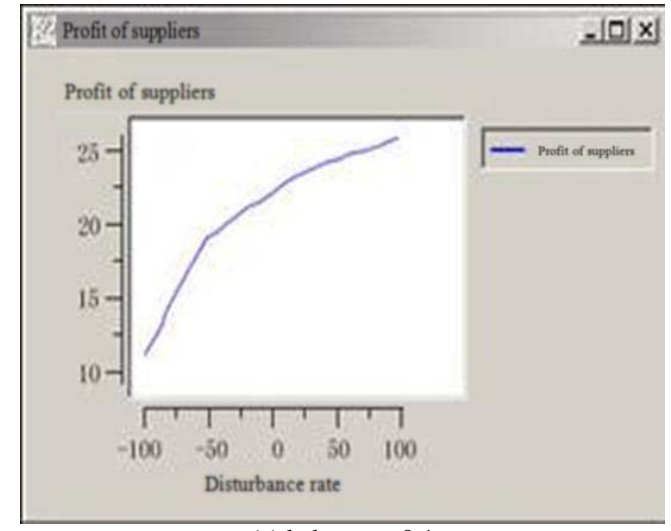

(a) kchange $=-0.1$

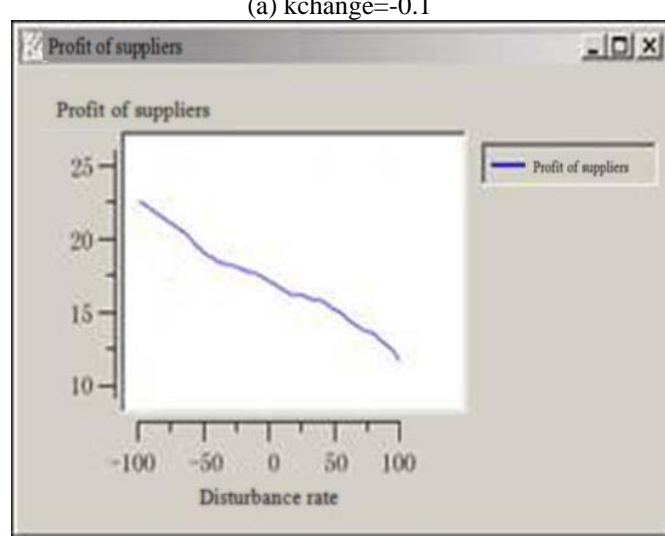

(b) kchange $=0.1$

Figure 3. Variation of profit of the supply chain after the disturbance

The impacts on the profits of supplier and retailer using emergency coordination strategy and maintaining the original strategy are shown in Fig.4 (a) and (b) respectively, where the market scale Dchange is in the range $[-100,100]$, when kchange is -0.1 or 0.1 .

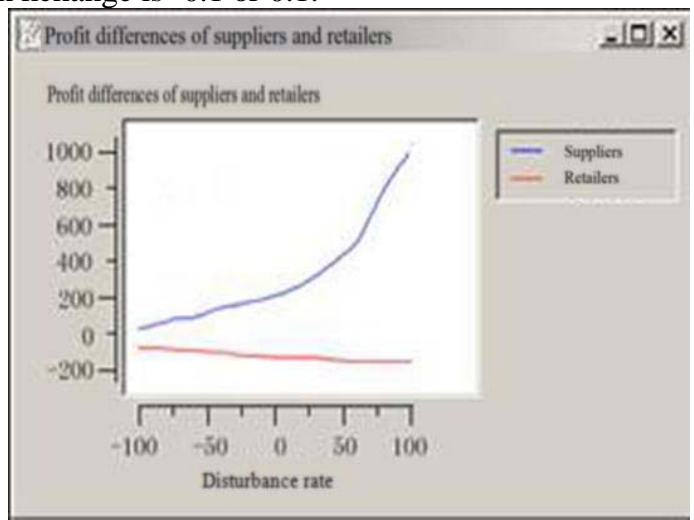

(a) kchange $=-0.1$

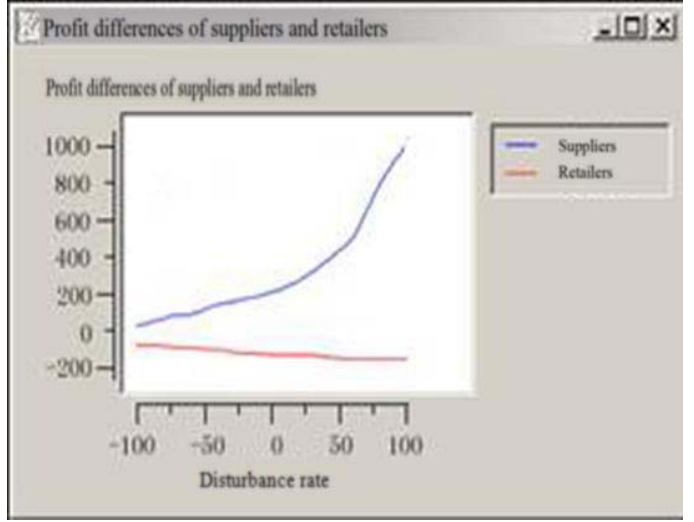

(b) kchange $=0.1$

Figure 4. The profit differences of suppliers and retailers after disturbance

Compared Fig.4 with Fig.2, it can be seen that the profit of the supplier increased when the optimal production plan unchanged or increased using the emergency coordination strategy. When the optimal production plan decreased, the profit of the supplier decreased using the emergency coordination strategy. It is just the opposite for the retailer.

\section{CONCLUSION}

In summary, when disturbances of the market scale and the price sensitive coefficient occurred simultaneously, the supplier should take emergency coordination strategy actively, which will not only increase the overall profit of the supply chain, but also can make itself do not suffer profit losses. At the same time, the economic phenomena are revealed that the retail price needs to make timely adjustment but the production plan does not need to change frequently when disturbance occurred. Therefore, after the impact on the supply chain of emergency disturbance, the appropriate emergency strategy to deal with the disturbance must be taken. It has an extremely far-reaching significance to the enterprises and to the economic development of the country.

\section{ACKNOWLEDGEMENTS}

The research work was supported by Natural Science Foundation of Shandong Province under Grant No. ZR2011GM008.

\section{REFERENCES}

[1] Yu hui, Chen jian, Yu gang. How to coordinate the supply chain inemergencies[J]. Systems Engineering-Theory and Practice (Chinese Edition), 7, pp.9-16, 2005.

[2] Latour A. A Trial by fire: a blaze in albuquerque sets-off major crisis for cell phone giants-Nokia handles supply shock with aploms as Ericsson gets burned, The Wall Street Journal, January 29, 2001.

[3] Wang chuantao, Ji shouwen, Shen jinsheng, Meng meng. Supply chain coordination disturbance cost and price sensitive coefficient [J], Supply Chain Management (Chinese Edition), 4, pp.91-93, 2009.

[4] Wu xiajin, Yang zehuan. Multi-retailer supply chain coordination with Cost and Demand Disruptions[J], Journal of Hunan University (NATURAL SCIENCE EDITION) (Chinese Edition), 5, pp.88-92, 2010 
[5] Yang zhihui, Chen hong, Zhao qian, Ma ronghua. Stochastic Demand and Production Cost at The Same Time under The Disturbance of Supply Chain Ripple effect [J], Journal of Management (Chinese Edition), 7(5), pp.728-732, 2010.
[6] Clausen J, Hansen J, Larsen J. Disruption management [J]. OR/MS Today, 28(5), pp.40-43, 2001. 